\title{
WIDESPREAD METABOLIC CHANGES ARE ASSOCIATED WITH LOW PRE- AND POST-ACTH STIMULATION CORTISOL IN DOGS
}

\section{Claudia Ottka1,2,3, Jenni Puurunen', Elisabeth Müller ${ }^{4}$, Corinna Weber ${ }^{4}$, Ruth Klein ${ }^{4}$, Hannes Lohi1,2,3 \\ ${ }^{1}$ PetBIOMICS Ltd, Helsinki, Finland, ${ }^{2}$ Department of Veterinary Biosciences and Department of Medical and Clinical Genetics, University of Helsinki, Helsinki, Finland, 3 Folkhälsan Research Center, Helsinki, Finland, ${ }^{4}$ LABOKLIN GmbH \& Co KG, Bad Kissingen, Germany *corresponding author, claudia.ottka@petbiomics.com}

\section{INTRODUCTION}

Hypoadrenocorticism can be diagnostically challenging. While it greatly affects metabolism, changes in the biochemical profile can be misleading, which can delay diagnostic testing, including $\mathrm{ACTH}$-stimulation.

\section{OBJECTIVES}

The aim of this study is to determine, what metabolic changes can be identified in serum samples of dogs with low pre- and post-ACTHstimulation cortisol values using quantitative nuclear magnetic resonance (NMR) metabolomics.

\section{METHODS}

- Cases $\mathrm{n}=36$ - pre- and postACTH stimulation cortisol $<20 \mathrm{ng} / \mathrm{ml}$

- Controls $\mathrm{n}=25$ - normal clinical chemistry

Canine-specific 'H-NMR metabolomics platform

\section{Z-score scaling}

Separate logistic regression models for each measurand

\section{Heatmap creation}

\section{RESULTS}

Controls

Cases

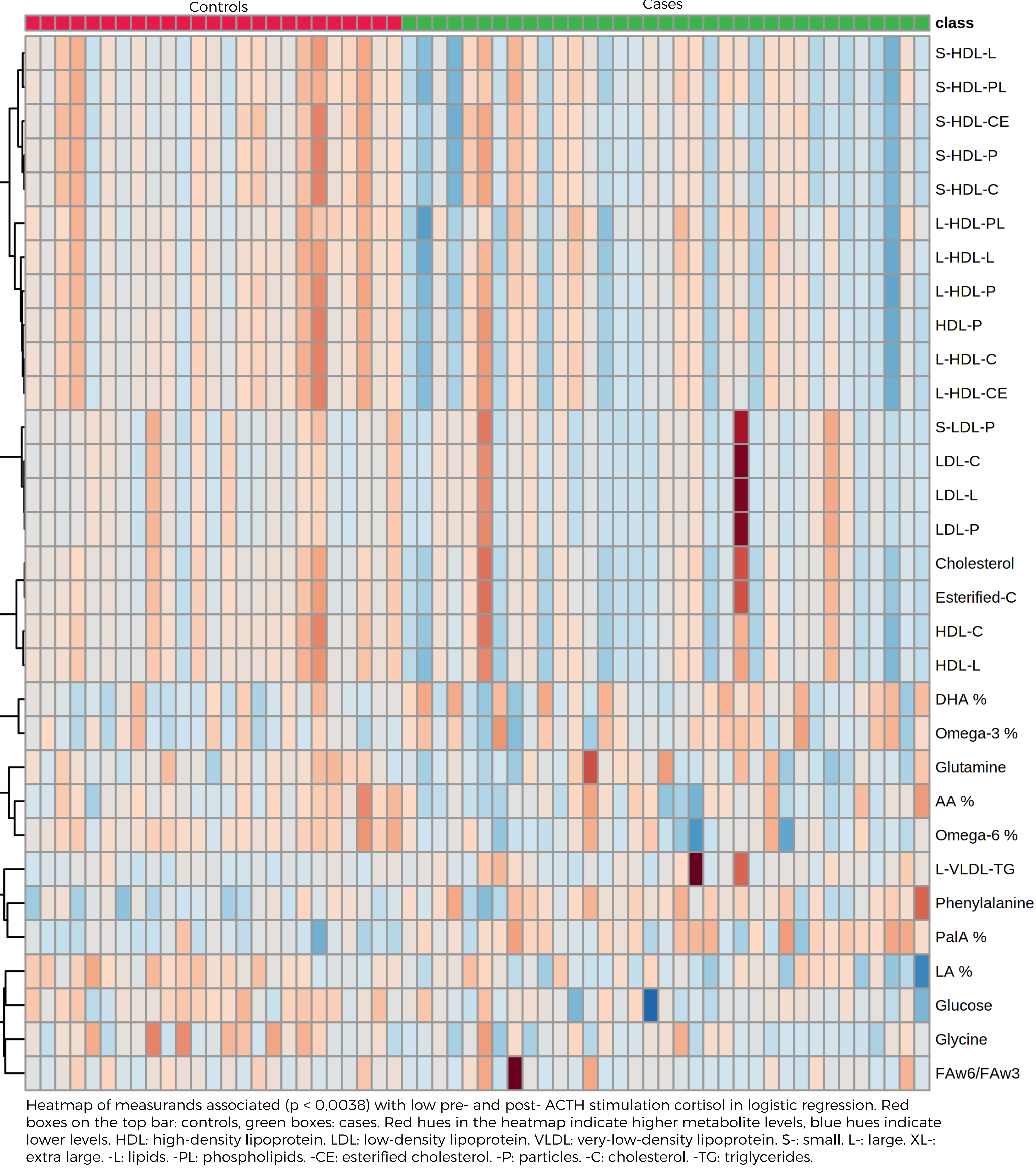

\section{CLINICAL SIGNIFICANCE}

Low cortisol levels associate with metabolic changes identifiable using NMR metabolomics. Lipoprotein subclass analysis may deepen our understanding on changes in cholesterol metabolism. The observed changes in amino acid concentrations may reflect changes in amino acid absorption, synthesis and protein catabolism. The high dispersion of metabolite values suggests, that also other factors than low cortisol concentrations contribute to the variation. Further studies are needed to associate these changes with clinical hypoadrenocorticism.

Reference

Ottka C, Puurunen J, Müller E, Weber C, Lo H. Metabolic changes associated with four

Acknowledgements
We thank Tuomas Poskiparta and Katja Jaun for help in project conceptualization and customers utilizing the services of LABOKLIN $\mathrm{CmbH} \& \mathrm{Co} \mathrm{KC}$ for enabling the scientific use PetBIOMICS LABOKLIN 